\title{
EFEITOS MULTIPLICADORES DO SETOR FLORESTAL NA ECONOMIA CAPIXABA ${ }^{1}$
}

\author{
Sebastião Renato Valverde ${ }^{2}$, Gilca Garcia de Oliveira ${ }^{3}$, Rosa Maria Armond Miranda Carvalho ${ }^{4}$ e \\ Thelma Shirlen Soares ${ }^{4}$
}

\begin{abstract}
RESUMO - Este estudo teve por objetivo avaliar a importância do setor florestal para o desenvolvimento social e econômico do Estado do Espírito Santo, por meio dos multiplicadores de impacto nos indicadores sociais e econômicos. Analisando dados do ano de 1999, observou-se que aumentos de $10 \%$ na produção florestal são suficientes para resultar na geração de mais 1.363 empregos formais no estado, de R $\$ 9,354$ milhões nas remunerações salariais, de R \$77,716 milhões no Produto Interno Bruto (PIB) e de R \$2 milhões na arrecadação de ICMS do estado, sendo aproximadamente $90 \%$ desses valores gerados dentro do próprio setor.
\end{abstract}

Palavras-chave: Economia florestal, planejamento florestal e matriz de contabilidade social.

\section{MULTIPLIER EFFECTS OF THE FOREST SECTOR ON THE ECONOMY OF THE STATE OF ESPÍRITO SANTO}

\begin{abstract}
The objective of this study was to evaluate the importance of the forest sector to the social and economical development of the State of Espírito Santo using impact multipliers on social and economical indicators. Analyzing data from 1999 it was observed that increases of 10\% in forest production is enough to result in the generation of more than 1.363 formal job positions in the State, $R \$ 9,354$ million in salaries, $R \$ 77,716$ million in Gross Domestic Product (GDP) and $R \$ 2$ million in ICMS collection for the State, with approximately $90 \%$ of these values being generated within the forestry sector itself.
\end{abstract}

Key words: Forestry economy, forestry planning and social accounting matrix.

\section{INTRODUÇÃO}

O interesse pelo tema deste trabalho surgiu a partir da percepção da necessidade de melhor compreender a importância da atividade florestal para o Estado do Espírito Santo, bem como sua contribuição para o desenvolvimento econômico estadual.

A cobertura florestal do território brasileiro, associada às excelentes condições edafoclimáticas para a silvicultura, confere ao país grandes vantagens comparativas para a atividade florestal.

A participação do setor florestal na economia do Brasil vem alcançando ampla mudança estrutural motivada, principalmente, em função da posição assumida pelo próprio governo brasileiro em relação à importância que o recurso florestal renovável possui para o adequado desenvolvimento do país.

Esse fato pode ser observado com maior facilidade se verificar que os resultados estatísticos apresentados

\footnotetext{
${ }^{1}$ Recebido em 10.12.2003 e aceito para publicação em 25.11.2004.

${ }^{2}$ Departamento de Engenharia Florestal da Universidade Federal de Viçosa. Viçosa, MG. E-mail: <valverde@ufv.br>.

${ }^{3}$ Departamento de Ciências Sociais Aplicadas à Agricultura da Universidade Federal da Bahia. Cruz das Almas, BA.

${ }^{4}$ Programa de Pós-Graduação em Ciência Florestal da Universidade Federal de Viçosa. Viçosa, MG.
} 
pelo setor florestal nas últimas décadas, principalmente aqueles referentes ao comércio exterior de produtos florestais, representam um indicador do nível de sustentação do desenvolvimento e da capacidade de processamento, diversificação e competitividade desse setor.

Ao examinar diferentes estudos do setor florestal brasileiro, pode-se observar sua importância para o país. Tal fato pode ser comprovado através da significativa participação do setor nos indicadores socioeconômicos, como no Produto Interno Bruto (PIB), na geração de empregos, na arrecadação de impostos, na remuneração do trabalho e na formação de divisas via exportações (VALVERDE et al., 2003).

Cabe, ainda, acrescentar uma particularidade desse setor: o emprego e a renda gerada ocorrem tanto no campo (trabalho florestal) quanto nas cidades (trabalho nas indústrias florestais), colaborando, assim, para a redução do êxodo rural (ALMEIDA, 2000).

Especificamente no Estado do Espírito Santo, o setor florestal merece destaque, pois o estado possui localização geográfica privilegiada e adequada infraestrutura de transportes marítimo, ferroviário e rodoviário (SCHETTINO et al., 2000). Entretanto, apesar de todas essas potencialidades e das perspectivas internacionais favoráveis ao setor florestal capixaba, principalmente a indústria de celulose, ainda tem que superar algumas barreiras de ordem política e econômica que ameaçam seu crescimento.

Nesse sentido, estudos que evidenciam as peculiaridades competitivas do setor florestal são importantes para elaboração de políticas que visem favorecer o desenvolvimento regional. Assim, o objetivo deste estudo foi determinar a participação dos efeitos multiplicadores do setor florestal empregando a análise da matriz de insumo-produto estimada para o Estado do Espírito Santo.

\section{MATERIAL E MÉTODOS}

\subsection{Fonte de dados}

Foram utilizados dados referentes ao ano de 1999, do Sistema de Contas Nacionais (SCN) do Instituto Brasileiro de Geografia e Estatística (IBGE), para as informações nacionais. As informações regionais foram coletadas das tabelas de valores de compra, venda, exportação e importação provenientes da Secretaria da Fazenda do Estado do Espírito Santo (SEFAZ-ES).
As atividades constantes da base de dados foram agregadas em 11 setores: agricultura (agropecuária); mineração (extrativa mineral, extração de petróleo e gás, minerais não-metálicos); metalsiderúrgico (siderurgia, metalurgia de não-ferrosos, outros metalúrgicos); maquinários (máquinas e tratores, material elétrico, equipamentos eletrônicos, automóveis, caminhões e ônibus, outros veículos e peças); florestal (madeira e mobiliário, papel e gráfica, indústria da borracha); plástico (artigos de plástico); petroquímicos (elementos químicos, refino do petróleo, químicos diversos, farmacêutica e de perfumaria); têxteis (indústria têxtil, artigos do vestuário, fabricação de calçados); alimentícios (indústria de café, beneficiamento de produtos vegetais, abate de animais, indústria de laticínios, indústria de açúcar, fabricação de óleos vegetais, outros produtos alimentares); diversos (indústrias diversas); e serviços (serviços industriais de utilidade pública, construção civil, comércio, transportes, comunicações, instituições financeiras, serviços prestados às famílias, serviços prestados às empresas, aluguel de imóveis, administração pública e serviços privados não-mercantis).

No entanto, vale esclarecer que a razão de se ter trabalhado com dados de 1999 foi devido ao fato de que esse foi o último ano em que as Tabelas de InsumoProduto do IBGE foram constituídas de informações observadas, pois a partir daí o que se tem são projeções feitas, principalmente em cima de variações no PIB.

\subsection{Matriz de multiplicadores de impacto}

Dentre os modelos econômicos empregados para estudos socioeconômicos, destacam-se os modelos computáveis de equilíbrio geral (CEG), os quais são capazes de simular o funcionamento de uma economia de mercado. Segundo Najberg et al. (1995), os CEGs caracterizam-se por permitir a substituição entre fatores e produtos na oferta e demanda, além de introduzir um sistema de preços de mercado e uma completa especificação dos fluxos de renda e produto.

Dos modelos econômicos de equilíbrio geral é possível obter os multiplicadores de impacto para saber quanto um setor influencia o restante da economia quando sofre aumentos na demanda de seus produtos. Esses impactos vão refletir em variações na renda, no PIB, nos empregos gerados, nos impostos arrecadados e na balança comercial (VALVERDE et al., 2003). 
Os fluxos intersetoriais de bens e serviços de uma economia, determinados por fatores tecnológicos e econômicos, podem ser descritos por um sistema de equações simultâneas, cuja relação matricial é dada por:

$$
\mathrm{X}=\mathrm{AX}+\mathrm{Y}
$$

em que:

$\mathrm{X}=$ vetor $(\mathrm{n} x \mathrm{1})$ representado pelo produto total de cada setor;

$\mathrm{Y}=\operatorname{vetor}(\mathrm{n} x \mathrm{1})$ representado pela demanda final setorial; e

$\mathrm{A}=$ matriz $(\mathrm{n} x \mathrm{n})$ representada pelos coeficientes técnicos de produção.

Nesse modelo, com o vetor de demanda final setorial conhecido e fixo, pode-se determinar o vetor de produto total setorial através da seguinte equação matricial:

$$
\mathrm{X}=(\mathrm{I}-\mathrm{A})^{-1} \mathrm{Y}
$$

A equação matricial (2) representa o modelo básico de Leontief, obtido da matriz de coeficientes técnicos de insumo-produto direta, sendo (I - A $)^{-1}$ a matriz dos multiplicadores de impacto de insumo-produto e Y, $o$ vetor de demanda final. Como no caso brasileiro o IBGE fornece as Tabelas Insumos-Produtos, separadas em tabela de consumo intermediário e tabela de produção, torna-se necessária a obtenção de uma matriz de coeficientes técnicos transformada. Para isso, adotou-se a tecnologia do setor com enfoque setor por setor, conforme o processo de transformação usado por Ramos (1997).

Neste estudo, a Matriz de Contabilidade Social (MCS) foi estruturada conforme a metodologia de Pyatt e Round (1985), considerando-se como bloco de contas endógenas atividades produtivas, instituições e valor adicionado e como blocos de conta exógena, conta, capital e resto do mundo, conforme sumarizado no Quadro 1.
Dessa forma, tem-se que:

$$
\mathrm{X}_{\mathrm{i}}=\mathrm{n}+\mathrm{Y}
$$

Da mesma forma, a renda recebida das contas exógenas é dada por:

$$
\mathrm{X}_{\mathrm{j}}=\mathrm{m}+\mathrm{z}
$$

Os elementos da matriz de transações endógenas $\left(\mathrm{T}_{\mathrm{ii}}\right)$ podem ser expressos como uma proporção da soma total, correspondente a cada coluna, isto é, como uma propensão média a gastar.

$$
\mathrm{T}_{\mathrm{ii}}=\mathrm{A}_{\mathrm{i}} \cdot \mathrm{X}_{\mathrm{i}}
$$

Sendo $\mathrm{X}_{\mathrm{i}}$ uma matriz diagonal, cujos elementos são $X_{i}(i=1, \ldots, n)$ e as colunas de $A_{i}$ representam as propensões médias a gastar. Similarmente:

$$
\mathrm{T}_{\mathrm{ji}}=\mathrm{A}_{\mathrm{k}} \mathrm{X}_{\mathrm{i}}
$$

Com a inclusão das matrizes $\mathrm{A}_{\mathrm{i}}$ e $\mathrm{A}_{\mathrm{k}}$, $\mathrm{n}$ e $\mathrm{m}$, descreve-se:

$$
\begin{aligned}
& \mathrm{n}=\mathrm{A}_{\mathrm{i}} \mathrm{X}_{\mathrm{i}} \\
& \mathrm{m}=\mathrm{AkXi}
\end{aligned}
$$

Em que $A_{i}$ e $A_{k}$ são, respectivamente, as matrizes de propensões médias a gastar das contas endógenas e exógenas. Assim, da expressão (3) obtém-se a equação básica da MCS, utilizada para o cálculo das suas matrizes de efeitos, conforme:

$$
\mathrm{X}_{\mathrm{i}}=\mathrm{A}_{\mathrm{i}} \mathrm{X}_{\mathrm{i}}+\mathrm{Y}
$$

tal que:

$\mathrm{Y}=\mathrm{X}_{\mathrm{i}}-\mathrm{A}_{\mathrm{i}} \mathrm{X}_{\mathrm{i}}$

\begin{tabular}{|c|c|c|c|c|c|c|}
\hline \multirow[b]{2}{*}{ Receitas } & \multirow[t]{2}{*}{ Despesas } & \multirow{2}{*}{$\begin{array}{c}\text { Contas } \\
\text { Endógenas }\end{array}$} & \multicolumn{3}{|c|}{ Contas } & \multirow[t]{2}{*}{ Total } \\
\hline & & & Soma & Exógenas & Soma & \\
\hline Contas Endógenas & & $\mathrm{T}_{\mathrm{ii}}$ & $\mathrm{N}$ & $\mathrm{T}_{\mathrm{ij}}$ & $\mathrm{Y}$ & $\mathrm{X}_{\mathrm{i}}$ \\
\hline Contas Exógenas & & $\mathrm{T}_{\mathrm{ji}}$ & M & $\mathrm{T}_{\mathrm{jj}}$ & $\mathrm{Z}$ & $X_{j}$ \\
\hline Total & & $\mathrm{X}_{\mathrm{i}}$ & & $\mathrm{X}_{\mathrm{j}}$ & & \\
\hline
\end{tabular}

ou seja,

$\mathrm{Y}=\left(\mathrm{I}-\mathrm{A}_{\mathrm{i}}\right) \mathrm{X}_{\mathrm{i}}$

Isolando X e usando a notação matricial, tem-se:

$\mathrm{X}=(\mathrm{I}-\mathrm{A})^{-1} \mathrm{Y}$

Quadro 1 - Representação simplificada das contas endógenas e exógenas da matriz de contabilidade social Table 1 - Simplified representation of the endogenous and exogenous bills of Social Accounting Matrix (SAM) 
Os valores da matriz A são determinados da seguinte forma:

$$
\begin{aligned}
& \mathrm{T}_{12}=\mathrm{A}_{12} \mathrm{X}_{2} ; \\
& \mathrm{T}_{22}=\mathrm{A}_{22} \mathrm{X}_{2} ; \\
& \mathrm{T}_{23}=\mathrm{A}_{23} \mathrm{X}_{3} ; \\
& \mathrm{T}_{31}=\mathrm{A}_{31} \mathrm{X}_{1} ; \mathrm{e} \\
& \mathrm{T}_{11}=\mathrm{A}_{11} \mathrm{X}_{1} .
\end{aligned}
$$

Desse modo, a matriz A é igual a:

$$
A=\left|\begin{array}{ccc}
A_{11} & A_{12} & 0 \\
0 & A_{22} & A_{23} \\
A_{31} & 0 & 0
\end{array}\right|
$$

Por meio das matrizes de coeficientes técnicos é possível verificar os impactos gerados quando ocorre estímulo ao desenvolvimento de um setor econômico via demandas exógenas devido às ligações intersetoriais que podem ser observadas pela análise dos multiplicadores de impacto do produto, da renda, do emprego, da arrecadação de impostos e do consumo de insumos produtivos, dentre outros.

O cálculo do multiplicador de dado setor econômico descreve a mudança que ocorre no produto total de todos os setores da economia resultante da mudança de uma unidade monetária da demanda final dos produtos de determinado setor.

Os multiplicadores econômicos resultantes da matriz de insumo-produto estimam o impacto global de variações na demanda final de um setor sobre uma variável econômica de interesse. Ou seja, os multiplicadores de impacto medem o efeito total na economia, em termos de geração de empregos, de impostos, remuneração ou valor agregado, dado um aumento em uma unidade de produto de uma indústria em particular. Portanto, com o aumento de uma unidade na demanda, ou seja, em Y1, o multiplicador para determinado setor é dado por:

$$
\left|\begin{array}{cccc}
A_{11} & A_{12} & \ldots & A_{1 n} \\
A_{21} & A_{22} & \ldots & A_{2 n} \\
\ldots & \ldots & \ldots & \ldots \\
A_{n 1} & A_{n 2} & \ldots & A_{n n}
\end{array}\right| \times\left|\begin{array}{c}
1 \\
0 \\
\ldots \\
0
\end{array}\right|=\left|\begin{array}{c}
A_{11} \\
A_{21} \\
\ldots \\
A_{n 1}
\end{array}\right|
$$

Na expressão anterior, o primeiro termo representa a matriz dos multiplicadores de impacto de insumoproduto e o segundo, o vetor de demanda final.

Os estímulos ao desenvolvimento de determinado setor desencadeiam uma série de impactos, de maneira que a variação na demanda de determinado produto de dado setor provoca mudanças na produção, na renda, no consumo de produtos importados, no recolhimento de impostos e no emprego.

As mudanças nos níveis das demais variáveis econômicas podem ser computadas pela multiplicação dos seus coeficientes técnicos pela variação na produção resultante do estímulo ao consumo setorial.

\subsection{Simulações no aumento do valor da produção do setor florestal capixaba}

De acordo com Brepohl (1980), os estímulos ao desenvolvimento de determinado setor desencadeiam uma série de impactos, de maneira que a variação na demanda de determinado produto de dado setor provoca mudanças na produção, na renda, no consumo de produtos importados, no recolhimento de impostos e no emprego.

As mudanças nos níveis das demais variáveis econômicas podem ser computadas pela multiplicação dos seus coeficientes técnicos pela variação na produção resultante do estímulo ao consumo setorial.

No caso das outras variáveis econômicas de interesse, como renda, emprego, impostos e importações, tem-se que uma mudança na necessidade de produto de determinado setor causa mudanças na produção, na renda, no consumo de produtos importados, no recolhimento de impostos e no emprego. Conforme descrito anteriormente, o impacto econômico na produção é estimado por:

$$
\Delta \mathrm{X}=(\mathrm{I}-\mathrm{A})^{-1} \cdot(\Delta \mathrm{Y})
$$

As mudanças nos níveis das outras variáveis econômicas são, então, calculadas pela multiplicação dos seus coeficientes técnicos pela variação na produção $(\Delta \mathrm{X})$ resultante da injeção no consumo setorial. Considerando, por exemplo, o impacto na renda, temse o como coeficiente de renda $r_{j}$ :

$$
r j=\frac{X_{s j}}{X_{j}}
$$


em que:

$$
\begin{aligned}
& \mathrm{r}_{\mathrm{j}}=\text { efeito direto da renda (coeficiente direto de } \\
& \text { renda); }
\end{aligned}
$$

$\mathrm{X}_{\mathrm{sj}}=$ quantidade do valor do trabalho ao setor j para produzir $X_{j}$; e

$X_{j}=$ valor bruto da produção do setor $\mathrm{j}(\mathrm{j}=1 \ldots . \mathrm{n})$.

Dessa forma, o multiplicador de impacto na renda $\left(\mathrm{R}_{\mathrm{I}}\right)$ é dado por $\mathrm{R}_{\mathrm{I}}=\mathrm{r}_{\mathrm{j}} \cdot \Delta \mathrm{X}$.

O mesmo raciocínio pode ser estendido para as outras variáveis (impostos, importações, emprego etc).

Para reforçar a análise da importância do setor florestal para o desenvolvimento do Estado do Espírito Santo, foram simulados aumentos no valor da produção desse setor, a fim de verificar o impacto nos indicadores socioeconômicos (PIB, emprego, salários e ICMS), em cada um dos 11 setores agregados e no total, através da relação intersetorial da cadeia produtiva.

Para essa simulação, foram utilizadas planilhas eletrônicas do programa computacional Excel, composto de todas as tabelas que constituíram a matriz de insumoproduto do Estado do Espírito Santo e das análises do modelo de Leontief, por meio dos multiplicadores de impacto do setor florestal.

Do ponto de vista prático, aumentos na produção do setor florestal capixaba podem ser representados pelo crescimento da indústria de celulose, o que vem ocorrendo desde a sua implantação. Na realidade, essa indústria vem apontando saltos enormes de crescimento na produção, tanto que no final de 2002 ela aumentou a produção de aproximadamente 1.262 milhão para 2 milhões de toneladas de celulose, quase $60 \%$ de crescimento. Considerando a predominância dessa indústria no total do setor florestal, isso significa que simulações através de aumentos porcentuais de até $50 \%$ no valor da produção do setor florestal capixaba são perfeitamente exeqüíveis.

Após o impacto da simulação na produção desse setor, verificou-se, pelo novo cenário, a posição no "ranking" da participação dos setores produtivos do estado, com o objetivo de mostrar a sensibilidade do setor em responder a aumentos na sua produção.

\section{RESULTADOS E DISCUSSÕES}

Utilizando a matriz de insumo-produto a preços básicos e aplicando os cálculos dos multiplicadores, obtêm-se os valores dos efeitos diretos que o aumento de uma unidade adicional na demanda final por produtos de cada setor causa na produção, nos salários, no emprego, nos impostos e nas importações, conforme mostrado no Quadro 2.

Os multiplicadores referem-se ao efeito que aumentos na demanda final por produtos de determinado setor produtivo irão provocar na economia como um todo, em termos de aumento na produção, no emprego, na renda, nos impostos e nas importações.

Quadro 2 - Multiplicadores de impacto na produção, nas importações, nos impostos, nos salários e no emprego do Estado do Espírito Santo, em 1999

Table 2 - Impact multipliers in products, imports, taxes, wages and employment of Espírito Santo State, in 1999

\begin{tabular}{lcccc}
\hline Setor & Produção & Importação & Impostos & Salários \\
\hline Agricultura & 1,269 & 0,002 & 0,005 & 0,007 \\
Alimentícios & 1,292 & 0,005 & 0,005 & 0,003 \\
Diversos & 1,166 & 0,008 & 0,005 & 0,014 \\
Florestal & 1,087 & 0,002 & 0,002 & 0,004 \\
Maquinário & 1,092 & 0,004 & 0,012 & 0,003 \\
Metal siderúrgico & 1,121 & 0,003 & 0,000 & 0,001 \\
Mineração & 1,147 & 0,005 & 0,002 & 0,017 \\
Petroquímico & 1,081 & 0,003 & 0,031 & 0,002 \\
Plástico & 1,247 & 0,003 & 0,005 & 0,004 \\
Serviços & 1,067 & 0,005 & 0,005 & 0,003 \\
Têxteis & 1,106 & 0,006 & 0,003 & 0,003 \\
\hline
\end{tabular}

Fonte: Elaborada pelos autores. 
No caso das importações, sabe-se que alguns setores, como agricultura, alimentícios e florestal, aumentam as suas produções, dado o crescimento da demanda de seus produtos, importando muito menos matéria-prima que outros, como veículo, eletroeletrônico e petroquímico. Dessa forma, considerou-se neste trabalho que, quanto menor o valor do coeficiente de impacto nas importações, melhor a contribuição do setor para o desenvolvimento econômico, pois aumentos nas importações podem contribuir para prejudicar a balança comercial.

No caso dos multiplicadores da produção, observou-se que os setores alimentício e agricultura foram os que apresentaram os maiores valores de impacto na produção, 1,292 e 1,269, respectivamente. No entanto, os setores que apresentaram as piores contribuições foram serviços $(1,067)$ e petroquímico $(1,081)$. O setor florestal exibiu o nono maior valor $(1,087)$, significando que, se a demanda final por produtos florestais aumenta $\mathrm{R} \$ 1.000,00$, ocorre variação global em todos os setores da economia para atender a essa mudança da ordem de $\mathrm{R} \$ 1.087,00$. Em outras palavras, $\mathrm{R} \$ 1.087,00$ indicam a quantidade de recursos necessários para adquirir os insumos requeridos pelo setor florestal para produzir $\mathrm{R} \$ 1.000,00$ do produto final desse setor, ou a mudança total na capacidade produtiva dos setores requeridos para atender ao estímulo exógeno por produtos florestais.

Os dados apresentados no Quadro 2 revelam que o setor florestal apresentou baixo efeito multiplicador na produção, o que não era esperado, porém se acredita que essa baixa "performance" ocorre porque o setor, no Estado do Espírito Santo, está sendo predominantemente representado por uma única indústria (no caso, a indústria de celulose).

Considerando apenas os efeitos diretos dos multiplicadores de impostos, renda, emprego e importações, pode ser destacado que o setor petroquímico apresenta as melhores contribuições para o desenvolvimento da economia do Estado do Espírito Santo, com a maior participação no recolhimento de impostos (0,031). Já o setor florestal aparece em décimo lugar, o que é devido ao fato de produzir produtos semi-elaborados e possuir carga tributária modesta, em comparação com produtos processados, de luxo ou supérfluos.
Quanto à geração de empregos, o multiplicador de empregos do setor florestal ficou em segundo lugar $(0,017)$ e o metalsiderúrgico, em primeiro $(0,036)$.

Comparado com os demais setores da economia capixaba, o setor florestal é o que menos provoca aumento nas importações de insumos quando aumenta a sua produção, dado o crescimento na demanda de seus produtos. Isso ocorre pela simples razão de a quase totalidade de seus insumos ser produzida domesticamente, o que é positivo para a balança de pagamentos da conta corrente da União, principalmente quando comparado com outros setores, em que grande parte de sua matériaprima é importada, como no caso das indústrias eletroeletrônicas, veículos, maquinários etc.

\subsection{Simulações no valor da produção}

Conforme o Quadro 3, variações mínimas de 10\% no valor da produção do setor florestal capixaba já são suficientes para gerar, no estado, 1.226 empregos, $\mathrm{R} \$ 8.681 .199,00$ de aumento nas remunerações salariais, $\mathrm{R} \$ 73.752 .713,00$ a mais no PIB e R $\$ 1.357 .233,00$ no recolhimento de ICMS, distribuídos proporcionalmente para cada setor, de acordo com a participação deles na cadeia produtiva do setor florestal.

Em todas as variáveis apresentadas no Quadro 3 , observa-se que a maior parte dos aumentos vai estar concentradas no setor florestal. No caso de emprego, $89,95 \%$ do total gerado será alocado no próprio setor, em seguida nos setores serviços, agricultura e petroquímico. Obviamente, incrementos na produção florestal, quer sejam aumentos na indústria de madeira e mobiliário (serrarias, fábricas de móveis e artefatos de madeira, usinas de tratamento de madeiras etc.), quer sejam na indústria de celulose, papel e gráfica e na indústria da borracha, vão gerar aumentos na demanda de matéria-prima, principalmente da madeira que virá de florestas tanto da própria indústria quanto de produtores rurais, mais provavelmente dos programas de fomento florestal, no caso do setor de agricultura.

No caso específico da indústria de celulose, aumentos na sua produção irão acarretar incremento no consumo de insumos químicos do processo produtivo, o que significa impacto no setor petroquímico e também aumentos em mais prestação de serviços para transporte de adicional produzido, comercialização, manutenção e reparos das máquinas, enfim, todo o aparato envolvido na cadeia de produção do setor florestal. 
Quadro 3 - Impacto do aumento no valor da produção do setor florestal, em 1999

Table 3 - Impact of the increase in the value of production of the forest sector, in 1999

\begin{tabular}{|c|c|c|c|c|c|c|c|c|}
\hline \multirow[t]{3}{*}{ Setor } & \multicolumn{8}{|c|}{ Porcentual de Aumento da Produção } \\
\hline & \multicolumn{4}{|c|}{$10 \%$} & \multicolumn{4}{|c|}{$50 \%$} \\
\hline & Emprego & Salários & PIB & ICMS & Emprego & Salários & PIB & ICMS \\
\hline Agricultura & 46 & 133.739 & 567.508 & 15.755 & 230 & 668.697 & 2.837 .540 & 78.774 \\
\hline Alimentícios & 1 & 4.012 & 54.269 & 3.890 & 7 & 20.060 & 271.343 & 19.449 \\
\hline Diversos & 8 & 18.121 & 37.366 & 2.679 & 40 & 90.604 & 186.831 & 13.395 \\
\hline Florestal & 1.226 & 8.681 .199 & 73.752 .713 & 1.357 .233 & 6.132 & 43.405 .997 & 368.763 .567 & 6.786 .166 \\
\hline Maquinários & 9 & 68.508 & 100.267 & 19.572 & 44 & 342.540 & 501.334 & 97.862 \\
\hline Metal siderúrgi & ico & 2.179 & 117.765 & 527 & 3 & 10.893 & 588.825 & 2.635 \\
\hline Mineração & 0 & 2.047 & 39.849 & 579 & 1 & 10.235 & 199.247 & 2.893 \\
\hline Petroquímico & 10 & 93.461 & 763.176 & 378.826 & 49 & 467.304 & 3.815 .878 & 1.894 .132 \\
\hline Plástico & 1 & 6.957 & 80.745 & 3.292 & 7 & 34.783 & 403.726 & 16.458 \\
\hline Serviços & 56 & 338.292 & 2.153 .962 & 162.930 & 279 & 1.691 .462 & 10.769 .811 & 814.649 \\
\hline Têxteis & 5 & 5.170 & 48.411 & 2.134 & 23 & 25.852 & 242.055 & 10.672 \\
\hline Variação total & 1.363 & 9.353 .686 & 77.716 .032 & 1.947 .417 & 6.814 & 46.768 .428 & 388.580 .159 & 9.737 .085 \\
\hline
\end{tabular}

Fonte: Elaborada pelos autores.

Acréscimos de 50\% no valor da produção do setor florestal são perfeitamente plausíveis, diante do aumento de $60 \%$ na produção de celulose da única indústria do estado, que passou a produzir 2 milhões de toneladas por ano a partir do final de 2002.

Considerando que no Estado do Espírito Santo a indústria de celulose representa $86,34 \%$ da participação do setor florestal na economia estadual, um acréscimo de $60 \%$ na produção $(60 \%$ x 86,34\% $=51,8 \%)$ influenciará positivamente a economia capixaba, segundo o Quadro
3, da seguinte maneira: geração de mais 6.814 empregos, remuneração de mais $\mathrm{R} \$ 46.768 .428,00$ em salários, incremento de mais $\mathrm{R} \$ 388.580 .159,00$ no PIB e recolhimento de $\mathrm{R} \$ 9.737 .085,00$ a mais de ICMS para os cofres do estado.

Verifica-se, no Quadro 4, que aumentos de 50\% já são suficientes para alterar a posição no "ranking" dos setores, como é o caso de emprego e ICMS, além de aproximar os valores das outras variáveis como os do setor imediatamente superior.

Quadro 4 - "Ranking” dos setores econômicos após aumentos de 10 e 50\% no valor da produção do setor florestal, em 1999

Table 4 -Ranking of the economic sections after increases of $10 \%$ and $50 \%$ in the value of the production of the forest sector, in 1999

\begin{tabular}{|c|c|c|c|c|c|}
\hline \multirow{13}{*}{ 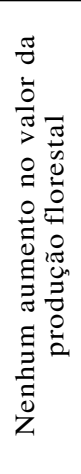 } & Setor & PIB & Empregos & Remunerações & ICMS \\
\hline & Agricultura & $89.009 .268(10)$ & $7.213(8)$ & $20.976 .000(9)$ & $2.471 .014(10)$ \\
\hline & Alimentícios & $643.715 .328(5)$ & $15.825(3)$ & $47.588 .113(5)$ & $46.139 .170(2)$ \\
\hline & Diversos & 14.009 .881 (11) & $3.016(10)$ & $6.794 .113(11)$ & $1.004 .453(11)$ \\
\hline & Florestal & 834.026 .014 (4) & $13.868(4)$ & $98.170 .573(2)$ & $15.348 .152(6)$ \\
\hline & Maquinários & 106.677 .457 & $9.445(5)$ & $72.888 .116(4)$ & 20.823 .738 (5) \\
\hline & Metalsiderúrgico & 1.783 .618 .678 & $7.915(7)$ & $32.997 .030(7)$ & $7.982 .939(8)$ \\
\hline & Mineração & 1.651 .852 .542 & $8.239(6)$ & $84.853 .000(3)$ & $23.983 .904(8)$ \\
\hline & Petroquímico & $315.609 .012(6)$ & $4.053(9)$ & $38.650 .457(6)$ & 156.662 .543 \\
\hline & Plástico & $132.960 .744(8)$ & $2.239(11)$ & $11.455 .236(10)$ & 5.420 .145 \\
\hline & Serviços & $16.471 .718 .048(1)$ & 427.209 (1) & $2.586 .980 .000(1)$ & 1.245 .952 .358 (1) \\
\hline & Têxteis & $235.239 .516(7)$ & $21.987(2)$ & 25.124 .362 & 10.371 .127 \\
\hline & Total & 22.278 .436 .489 & 521.010 & 3.026 .477 .000 & 1.536 .159 .542 \\
\hline
\end{tabular}


Quadro 4-Cont.

Table 4 - Cont.

\begin{tabular}{|c|c|c|c|c|c|}
\hline \multirow{13}{*}{ 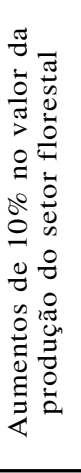 } & Setor & PIB & Empregos & Remunerações & ICMS \\
\hline & Agricultura & $89.576 .776(10)$ & $7.259(8)$ & $21.109 .739(9)$ & $1.007 .132(11)$ \\
\hline & Alimentícios & $643.769 .597(5)$ & $15.827(3)$ & $47.592 .124(5)$ & 23.984 .482 \\
\hline & Diversos & 14.047 .247 (11) & $3.024(10)$ & $6.812 .234(11)$ & 134.950 .302 (2) \\
\hline & Florestal & 907.778 .727 (4) & $15.095(4)$ & $106.851 .773(2)$ & 10.373 .261 \\
\hline & Maquinários & 106.777.724 (9) & $9.454(5)$ & $72.956 .624(4)$ & $16.705 .385(6)$ \\
\hline & Metalsiderúrgico & $1.783 .736 .443(2)$ & $7.915(7)$ & $32.999 .208(7)$ & $5.423 .437(9)$ \\
\hline & Mineração & 1.651 .892 .391 & $8.239(6)$ & 84.855 .047 (3) & $20.843 .310(5)$ \\
\hline & Petroquímico & $316.372 .188(6)$ & $4.063(9)$ & $38.743 .918(6)$ & 46.143 .060 (3) \\
\hline & Plástico & $133.041 .489(8)$ & $2.241(11)$ & $11.462 .193(10)$ & $2.486 .769(10)$ \\
\hline & Serviços & 16.473 .872 .010 & 427.265 (1) & $2.587 .318 .292(1)$ & 157.041 .369 (1) \\
\hline & Têxteis & 235.287 .928 (7) & $21.992(2)$ & $25.129 .532(8)$ & $7.983 .466(8)$ \\
\hline & Total & 22.356 .152 .521 & 522.373 & 3.035 .830 .686 & 1.538 .106 .959 \\
\hline \multirow{12}{*}{ 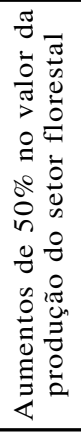 } & Agricultura & $91.846 .808(10)$ & $7.443(8)$ & $21.644 .697(9)$ & $2.549 .788(10)$ \\
\hline & Alimentícios & $643.986 .671(5)$ & $15.832(4)$ & $47.608 .172(5)$ & 46.158 .619 (3) \\
\hline & Diversos & $14.196 .712(11)$ & $3.057(10)$ & $6.884 .717(11)$ & $1.017 .848(11)$ \\
\hline & Florestal & 1.202 .789 .581 (4) & $20.000(3)$ & 141.576 .571 & $22.134 .318(5)$ \\
\hline & Maquinários & 107.178.791 (9) & $9.489(5)$ & 73.230 .655 (4) & $20.921 .600(6)$ \\
\hline & Metalsiderúrgico & 1.784 .207 .503 & 7.917 (7) & $33.007 .923(7)$ & $7.985 .574(8)$ \\
\hline & Mineração & 1.652 .051 .789 & $8.240(6)$ & 84.863 .235 (3) & 23.986 .797 (4) \\
\hline & Petroquímico & $319.424 .891(6)$ & $4.102(9)$ & $39.117 .762(6)$ & 158.556 .675 (2) \\
\hline & Plástico & $133.364 .470(8)$ & $2.246(11)$ & $11.490 .019(10)$ & $5.436 .603(9)$ \\
\hline & Serviços & 16.482 .487 .859 & 427.488 (1) & $2.588 .671 .462(1)$ & $1.246 .767 .007(1)$ \\
\hline & Têxteis & $235.481 .572(7)$ & $22.010(2)$ & $25.150 .214(8)$ & $10.381 .799(7)$ \\
\hline & Total & 22.667 .016 .648 & 527.824 & 3.073 .245 .428 & 1.545 .896 .628 \\
\hline
\end{tabular}

\section{CONCLUSÕES}

De acordo com os resultados neste estudo, concluiuse que o setor florestal contribui para o desenvolvimento socioeconômico do Estado do Espírito Santo, tanto na participação da formação desses indicadores (PIB, geração de empregos, remunerações salariais, balança comercial e arrecadação de ICMS) quanto no impacto de seu crescimento, quando há aumento na demanda da economia.

Além dessa participação, o setor apresenta uma "performance" favorável nos multiplicadores de impactos desses indicadores, principalmente no que tange a empregos e salários, devido à natureza das suas indústrias que são, na sua maioria, intensivas em mãode-obra, com uma vantagem adicional de que esses empregos são gerados tanto no campo quanto nas cidades, contribuindo para a redução do desemprego e do êxodo rural.

\section{REFERÊNCIAS BIBLIOGRÁFICAS}

ALMEIDA, A. R. C. Gestão operacional da qualidade: uma abordagem prática e abrangente no setor florestal. Campinas: UNICAMP, 2000. 128 p.

BREPOHL, D. Custos em empreendimentos florestais. Curitiba: FUPEF, 1980. 18 p. (Série técnica, 4)

CARVALHO, R. M. M. et al. O papel do setor florestal brasileiro no contexto nacional. In: CONGRESSO FLORESTAL BRASILEIRO, 8., 2003, São Paulo. Anais... São Paulo: SBS/SBEF, 2003. v. 2. CD-ROM.

NAJBERG, S.; RIGOLON, F. J. Z.; VIEIRA, S. P. Modelo de equilíbrio geral computável como instrumento de política econômica: uma análise de câmbio versus tarifas. Rio de Janeiro: BNDES, 1995. 33p. (Texto para discussão, 30). 
PYATT, G.; ROUND, J. I. Social accounting matrices for development planning. In: PYATT, G.; ROUND, J. I. Social accounting matrices: a basis for planning. Washington: World Bank, 1985. p. 52-69.

RAMOS, R. L. O. Uma comparação dos modelos para o cálculo dos

coeficientes técnicos diretos de

Leontief. Rio de Janeiro: COPPE/UFRJ, 1997. 253p.
SCHETTINO, L. F. et al. Diagnóstico para a gestão florestal sustentável no Espírito Santo. Revista Árvore, v.24, n.4, p.445456, 2000.

VALVERDE, S. R. et al. Efeitos multiplicadores da economia florestal brasileira. Revista Árvore, v.27, n.3, p.285-293, 2003. 
